$93,75 \%$ và $100 \%$, trong khi đó với ổ dị dạng có kích thước nhỏ thì tỷ lệ phát hiện thấp hơn với 40,9\%. So sánh tốc độ dòng chảy bên nuôi ổ dị dạng với bên đối diện, chỉ có động mạch não giữa có tốc độ tăng lớn hơn có ý nghĩa thống kê với $p<0,05$. Theo nghiên cứu của Phan Văn Đức [4] về độ nhạy và độ đặc hiệu tính theo kích thước ổ dị dạng thì nhóm có kích thước trung bình và lớn có độ nhạy cao (thấp nhất là $87,5 \%$ cao nhất là 100\%). Mặt khác, với nhóm kích thước nhỏ thì xấp xỉ 50\% là không phát hiện được trên siêu âm xuyên sọ. Kết quả này của chúng tôi cũng gần tương tự với kết quả của Mast và cộng sự [8] khi nghiên cứu 114 bệnh nhân AVM với siêu âm Doppler xuyên sọ có độ nhạy của tốc độ trung bình và chỉ số mạch lần lượt là $85 \%$ và $63 \%$ còn khi phân nhóm thì nhóm kích thước trung bình và lớn cũng có độ nhay khá cao (từ 73 đến 100\%) còn nhóm kích thước nhỏ thì có độ nhạy khá thấp 39\% và $31 \%$ lần lượt theo tốc độ trung bình và chỉ số mạch. Về độ đặc hiệu có giá trị là $96 \%$ và $100 \%$ lần lượt theo tốc độ dòng chảy và chỉ số mạch.

\section{KẾT LUẬN}

Độ tuổi hay gặp nhất của các bệnh nhân AVM võ là từ trên 40 tuổi chiếm tỷ lệ $63,9 \%$; tuổi trung bình $43 \pm 14,7$. Bệnh gặp ở cả hai giới với tỷ lệ nam/nữ là 1,27/1. Chảy máu do võ AVM là chảy máu thùy chiếm $85,72 \%$. Ổ dị dạng võ thường là ổ có kích thước nhỏ $<3 \mathrm{~cm}$, có một tĩnh mạch dẫn lưu duy nhất. TCD là phương pháp hữu ích để chẩn đoán các dị dạng thông động tĩnh mạch não nó có độ nhạy cao với các AVM có kích thước trung bình.

\section{TÀI LIÊU THAM KHẢO}

1. Friedlander RM. Arteriovenous malfor- mations of the brain. N Engl J Med 2007; 356:2704-12.

2. Cognard C, Spelle L., and Pierot L. (2004), Pial arteriovenous malformations in: Intracranial vascular malformations and aneurysm, Springer. 39-92.

3. Shaligram S.S., Winkler E., Cooke $D$. và cộng sự. (2019). Risk factors for hemorrhage of brain arteriovenous malformation. CNS Neurosci Ther, 25(10), 1085-1095.

4. Phan 'Văn Đức, Lê Văn Thính, Hoàng Văn Thuận (2018), siêu âm Doppler xuyên so và hình ảnh chụp mạch máu não của dị dạng thông động-tĩnh mạch nã̃o.

5. Marco A.Stefani, Phillip J.Porter, et al (2002), Large and deep brain arteriovenous malformation are associated with risk of future hemorrhage, Stroke, 3. 1220.

6. Deruty $R$, et al (1985), Les malformations Arterio-veineuses Cerebrales, Neurochir, 31. 21-29

7. Pham Hông Đức, Pham Minh Thông, Lê Văn Thính (2010), Các yễu tố cấu trúc mạch liên quan đên biểu hiện xuất huyết của dị dạng động tînh mach não, Tạp chí $Y$ học thực hành (705) số 2, 52-55.

8. Mast H, Mohr JP, Osipov A, et al (1995) Steal is an unestablished mechanism for the clinical presentation of cerebral arteriovenous malformations, Stroke, 26. 1215-1220

\title{
GIÁ TRI CỦA XUNG KHUẾCH TÁN TRONG UNG THƯ TUYẾN TIỀN LIÊTT: VÙNG NGOẠI VI VÀ VÙNG CHUYỂN TIẾP
}

\section{TÓM TẮT}

Mục tiêu : Đánh giá giá trị của chuỗi xung khuếch tán (DWI) trong chấn đoán ung thư tuyến tiền liẹt (UTTTL) ở vùng ngoại vi (NV) và vùng chuyển tiểp (CT). Phương tiện và phương pháp: Chúng tôi thu thập số liệu của 74 bệnh nhân gồm 296 tổn thương đã được chụp cộng hưởng từ (CHT) tuyến tiền liệt (TTL) 3.0 tesla và đã có kết quả mô bênh học. Tổn thương nghi ngờ UTTTL được phân độ bằng phân độ PIRADs cho chuỗi xung DWI theo PIRADs 2.1 từ đó phân tích mối tương quan giữa hình đặc điểm hình ảnh trên

${ }^{1}$ Trường Đại họ Y Hà Nội

${ }^{2}$ Bênh viên Hữu Nghi Việt Đức

Chịu trách nhiệm chính: Nguyễn Thị Hải Anh

Email: haianhnguyen.918@gmail.com

Ngày nhận bài: 2.6.2021

Ngày phản biên khoa hoc: 29.7.2021

Ngày duyệt bài: 9.8.2021

\section{Nguyễn Thị Hải Anh ${ }^{1}$, Nguyễn Duy Hùng ${ }^{1,2}$}

DWI và mô bênh hoc của tổn thương. Kết quả: Trong số 74 bênh nhân với 296 tổn thương, $182(61,5 \%)$ tổn thương nghi ngờ trên DWI (42,8\% ở NV; $57,2 \%$ ở CT). Phân tích hồi quy chỉ ra có mối liên quan đáng chú ý giữa độ DWI cao và mức độ nă̆ng trên mô bệnh học thể hiện bằng điểm Gleason (tất cả có $p<0,05$ ). Tỉ lệ tương quan giữa độ DWI và kết quả mô bệnh học là $77,0 \%$, NV cao hởn CT $(87,8 \%$ và $69,9 \%$ với $p=$ $0,006)$. Kết quả này chứng minh có mối tương quan tuyến tính giữa sự tăng lên độ DWI và mức độ ác tính của tổn thương. Kết luận: Trong xác định ung thư, DWI chính xác hơn ở vùng ngoại vi so với vùng chuyển tiếp. Phân độ DWI càng cao thì độ chính xác càng cao. Trong khi đó, UTTTL có thể tìm thây ở một phần nhỏ case có DWI âm tính.

Tư khóa: ung thư tuyến tiền liêt, xung khuếch tán DWI, PIRADs, cộng hưởng từ tuyến tiền liệt.

\section{SUMMARY}

VALUE OF DIFFUSION-WEIGHTED 


\section{IMAGING IN DIAGNOSIS OF PROSTATE CANCER IN THE PERIPHERAL ZONE AND TRANSITION ZONE}

Objectives: Evaluation of the value of diffusion weighted imaging (DWI) in the diagnosis of prostate cancer in the peripheral zone and the transition zone. Methods and subject: We reviewed the data of 74 patients including 296 lesions, who underwent magnetic resonance imaging of the prostate and had histopathological results. Suspected lesions were graded by PIRADs for DWI, then, the correlation between the imaging features on DWI and the histopathology of the lesion have DWI grade $\geq$ III would be analyzed. Results: Among 74 patients with 296 lesions, $182(61.5 \%)$ suspected lesions on DWI (42.8\% in peripheral zone, $57.2 \%$ in transition zone). Regression analysis showed a remarkable association between high DWI grade and the adverse pathological prognosis expressed by Gleason grade (all with $p<$ $0.05)$. The concordance rate between DWI grade and histopathology was $77 \%$, NV was remarkably higher than CT $(87.8 \%$ and $69.9 \%$ with $\mathrm{p}=0.006)$. This result proved that there is a correlation between the increase in DWI grade and the malignancy of the lesion. Conclusions: In detecting tumors, DWI is more accurate in the peripheral zone than in the transition zone. The higher the DWI grade, the higher the accuracy. Meanwhile, prostate cancer could be found in a small proportion of negative DWI cases.

Keywords: prostate cancer, diffusion weighted imaging, PIRADs, magnetic resonance imaging.

\section{I. ĐĂT VẤN ĐỀ}

Cộng hưởng từ $(\mathrm{CHT})$ đa chuỗi xung là phương tiện để chẩn đoán chính xác và giúp phân độ ung thư tuyến tiền liệt (UTTTL). Gần đây, Hiệp hội chẩn đoán hình ảnh niệu-sinh dục châu Âu khuyến cáo sử dụng CHT đa chuỗi xung như là phương pháp chẩn đoán hình ảnh tiêu chuẩn cho đánh giá tuyến tiền liệt ${ }^{1}$. Nhiều nghiên cứu chỉ ra rằng $\mathrm{CHT}$ đa chuỗi xung có thể xác định chính xác những u khu trú, cho giá trị dự đoán âm tính cao trong xác định UTTTL2

Chuỗi xung khuếch tán (DWI) là một chuỗi xung quan trọng và đem lại nhiêu giá trị trong CHT tuyến tiền liệt đặc biệt trong xác định và phân độ UTTTL ${ }^{1}$. DWI có thời gian chụp ngắn, độ phân giải cao giữa tổn thương lành tính và ác tính. Bản đồ hệ số khếch tán (ADC) giúp ích cho phân độ của u và ước tính mức độ u. Mă̆t khác, xung DWI cũng có thể bị ảnh hưởng bởi nhiều yếu tố nhiễu ${ }^{3}$. Mặc dù vậy, phương pháp tối ưu cho đánh giá $A D C$ chưa được xác định. Cho dù DWI được đánh giá là phương pháp đánh giá tổn thương vùng ngoại vi (NV) có độ chính xác cao, mức độ hiệu quả của DWI trong đánh giá tổn thương vùng chuyển tiếp $(C T)$ còn bàn cãi ${ }^{4}$. Chẩn đoán $u$ vùng $C T$ bằng $T 2 W$ chỉ bị hạn chế bởi tín hiệu không đồng nhất ở bệnh nhân nam lớn tuổi, phối hợp DWI và T2W được chỉ ra là tăng giá trị chẩn đoán. The phân loại PIRADs phiên bản 2.1, DWI là xung đầu tay cho đánh giá vùng NV chứ không phải $\mathrm{CT}^{1}$. Trong nghiên cứu này, chúng tôi đánh giá và so sánh độ chính xác của DWI, một xung quan trọng của CHT thăm khám TTL, trong xác định UTTTL ở NV và $\mathrm{CT}$.

\section{II. ĐỐI TƯƠ'NG VÀ PHƯƠNG PHÁP NGHIÊN CỨU}

Kết quả của 74 bênh nhân đã được chụp $\mathrm{CHT}$ trước sinh thiết từ tháng 7 năm 2020 đên tháng 3 năm 2021 và có kết quả giải phẫu bệnh. Hình ảnh $\mathrm{CHT}$ được chụp bằng máy 3.0 tesla (GE, Healthcare, II, Hoa Kỳ), sử dụng coil toàn thân. Ảnh DWI được chụp với các thông số thời gian lặp lại 3000ms, trường chụp 220x220mm, độ dày lát cắt $3 \mathrm{~mm}$, khoảng cách lát cắt $1 \mathrm{~mm}$, ma trận $288 \times 288$. Gradient mã hóa xung khuếch tán được chụp với hai giá trị b0 và b1500 s/mm². Bác sĩ chẩn đoán hình ảnh được làm mù với kết quả giải phẫu bênh, đọc kết quả $\mathrm{CHT}$ trên hê thống INFINITT PACs (Infinitt healthcare, Seoul, South Korea). Mỗi bệnh nhân sẽ được phân thành 4 mẫu tổn thương tương ứng với vùng ngoại vi phải, vùng chuyển tiếp phải, vùng ngoại vi trái và vùng chuyển tiếp trái. Tổn thương nghi ngờ nhất của mỗi vùng được phân độ 5 độ theo phân loại PIRADs 2.1 cho chuối xung DWI, Phân độ tổn thương trên $\mathrm{CHT}$ được tính một cách hệ thống dựa trên các đặc điểm như kích thước (đo trên ảnh $T 2 W$ ), hình thái và tín hiệu của tổn thương trên DWI. Cu thể, DWI III là tổn thương khu trú giảm tín hiệu rõ trên $A D C$ hoặc tăng rõ tín hiệu trên DWI $b$ cao nhưng không đồng thời cả hai, DWI IV là tổn thương khu trú giảm rõ trên $A D C$ và tăng rõ trên DWI $b$ cao, kích thước chiều lớn nhất $<1,5 \mathrm{~cm}$, DWI $V$ là tổn thương khu trú giảm rõ trên $A D C$ và tăng rõ trên DWI $b$ cao, kích thước chiều lớn nhất $\geq 1,5 \mathrm{~cm}$ (minh họa hình 1).

Chỉ số mô bênh học được tính theo tổn thương có điểm Gleason cao nhất và/ hoặc lớn nhất. Khi bênh nhân có điểm DWI $\leq$ II có thể không có chỉ số tổn thương, chỉ những bệnh nhân có điểm DWI $\geq$ III được phân tích tương quan giữa chỉ số mô bệnh học và chỉ số trên hình ảnh. Khi vị trí vùng của mảnh sinh thiết tương ứng với vị trí tổn thương nghi ngờ trên $\mathrm{CHT}$, mối tương quan sẽ được đánh giá. Chẩn đoán UTTTL phụ thuộc và kết quả giải phẫu bệnh, UTTTL độ cao trên mô bệnh học được đưa ra khi điểm Gleason $\geq 4+3$.

Test Chi-square và student $T$ tests được dùng để so sánh đặc điểm giữa các nhóm. Tỷ suất chênh (OR) được dùng để đánh giá mối tương 
quan giữa đặc điểm DWI và tiên lượng mô bệnh học. Tất cả các phân tích được sử dụng SPSS
20.0 (Chicago, IL, USA). Tất cả các giá trị p đo 2 phía và $p<0,05$ được coi là có ý nghĩa thống kê.
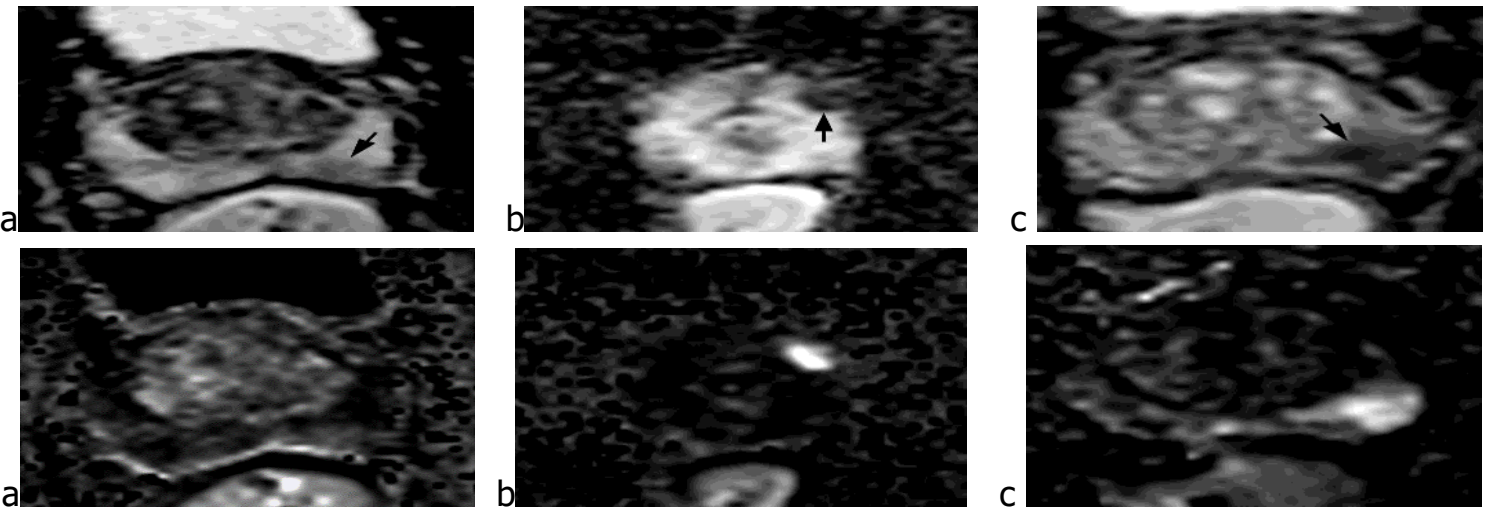

Hình 1. Minh họa hình ảnh tôn thương trên chuỗi xung khuếch tán (DWI) theo phân độ PIRADs'.

a. Độ III: Tổn thương khu trú (mũi tên) giảm tín hiệu rõ trên $A D C$ hoặc tăng rõ tín hiệu trên DWI b cao nhưng không đồng thời cả hai

b.Độ IV: Ổ tổn thương khu trú giảm rõ trên $A D C$ và tăng rõ trên DWI b cao, kích thước chiều lớn nhất $<1,5 \mathrm{~cm}$.

c Độ V: Ổ tổn thương khu trú giảm rõ trên $A D C$ và tăng rõ trên DWI b cao, kích thước chiều lớn nhất $\geq 1,5 \mathrm{~cm}$.

\section{KẾT QUẢ NGHIÊN CỨU}

Kết quả giải phẫu bệnh của bệnh nhân được thể hiện trong bảng 1. Có 114 (38,5\%) mẩu không có tổn thương nghi ngờ trên MRI và $182(61,5 \%)$ mẫu có tổn thương nghi ngờ (độ DWI $\geq 3$ ). Trong số 182 mẫu có có 76 mẫu là tổn thương ở NV và 105 mẫu ở $\mathrm{CT}$. Những bệnh có tổn thương nghi ngờ có những đặc điểm đáng chú ý bao gồm tuổi cao hơn và thể tích tuyến tiền liệt thấp hơn. Những phân tích hồi quy chỉ ra có sự liên quan giữa độ DWI cao hơn và mức độ nặng trên giải phẫu bệnh thể hiện bởi điểm Gleason cao hơn ( $p<0,05)$ (Bảng 2).

Bảng 1. Đặc điểm lâm sàng-mô bệnh học của các đôî tượng nghiên cứu theo mức độ nghi ngờ của tổn thương trên DWI.

\begin{tabular}{|c|c|c|c|}
\hline & $\begin{array}{c}\text { Tổn thương } \\
\text { không nghi } \\
\text { ngờ (n=114) }\end{array}$ & $\begin{array}{c}\text { Tốn thương } \\
\text { nghi ngờ } \\
\text { (n=182) }\end{array}$ & $\begin{array}{c}\text { Giá } \\
\text { trị p }\end{array}$ \\
\hline $\begin{array}{c}\text { Tuối } \\
\text { (năm) }\end{array}$ & $\begin{array}{c}66 \\
(53-80)\end{array}$ & $\begin{array}{c}70 \\
(51-88)\end{array}$ & $<0,001$ \\
\hline $\begin{array}{c}\text { Thể tích } \\
\text { tuyến } \\
\text { tiền liệt }\end{array}$ & $59,30(21-116)$ & $\begin{array}{c}49,19 \\
(16-156)\end{array}$ & 0,03 \\
\hline $\begin{array}{c}\text { Điếm } \\
\text { Gleason }\end{array}$ & & & $<0,001$ \\
\hline$\leq 7$ & $106(93,0 \%)$ & $66(36,3 \%)$ & \\
\hline$=8$ & $1(0,9 \%)$ & $32(17,6 \%)$ & \\
\hline
\end{tabular}

\begin{tabular}{|c|c|c|c|}
\hline$\geq 9$ & $7(6,1 \%)$ & $84(46,2 \%)$ & \\
\hline Độ DWI & & & $<0,001$ \\
\hline Độ I-II & $114(100 \%)$ & & \\
\hline Độ III-IV & & $72(39,6 \%)$ & \\
\hline Độ V & & $110(60,4 \%)$ & \\
\hline \multicolumn{3}{|c|}{ Điểm Gleason } & $<0,001$ \\
\hline$\leq 3+4$ & $103(90,4 \%)$ & $57(31,3 \%)$ & \\
\hline$=3+4$ & $3(2,6 \%)$ & $9(4,9 \%)$ & \\
\hline$\geq 4+4$ & $8(7,0 \%)$ & $116(63,7 \%)$ & \\
\hline $\begin{array}{c}\text { Xâm lấn } \\
\text { vỏ bao }\end{array}$ & $4(12,5 \%)$ & $\begin{array}{c}112 \\
(42,4 \%)\end{array}$ & 0,14 \\
\hline
\end{tabular}

Bảng 2. Phân tích hôi quy môi liên quan độ DWI với mô bệnh học.

\begin{tabular}{|c|c|c|c|c|}
\hline $\begin{array}{c}\text { Điếm } \\
\text { cuối }\end{array}$ & \multicolumn{4}{|c|}{ Toàn bộ bệnh nhân } \\
\hline & $\begin{array}{c}\text { Mức độ } \\
\text { nghi ngờ }\end{array}$ & OR & $\begin{array}{c}\text { Khoảng } \\
\mathbf{9 5 \%} \text { CI }\end{array}$ & $\begin{array}{c}\text { Giá } \\
\text { trị p }\end{array}$ \\
\hline $\begin{array}{c}\text { pGS } \geq \\
4+3\end{array}$ & I-II & \multicolumn{3}{|c|}{ Đối chứng } \\
\hline & III-IV & 1,382 & $\begin{array}{c}1,163- \\
1,641\end{array}$ & $<0,001$ \\
\hline & $\mathrm{V}$ & 2,980 & $\begin{array}{c}2,242- \\
3,961\end{array}$ & $<0,001$ \\
\hline
\end{tabular}

Tỉ lệ tương quan toàn bộ giữa độ tổn thương trên hình ảnh và tổn thương mô học chỉ ra sự tuyến tính dương với sự tăng lên của độ DWI. (độ III : 30\%, độ IV 91,7\%, độ V 98,1\% với $p$ $<0,05)$. Khi chúng tôi so sánh tỉ lệ tương quan giữa NV và $\mathrm{CT}$, NV cho thấy có tỉ lệ tương quan cao hơn CT (87,0\% với 69,0\%, $\mathrm{p}=0$ 0,006) (Bảng 3). Tuy nhiên, phân tích dưới nhóm chỉ ra rằng sự khác biệt này chỉ có ý nghĩa với tổn thương độ IV, V (độ V cho kết quả NV 98,1\% so với CT $83,3 \%, p=0,02)$. Không có sự khác biệt đáng kể giữa các dưới nhóm ở bệnh nhân có tổn thương độ III (tất cả giá trị $\mathrm{p}<0,05$ ). Tỉ lệ mẫu có độ mô học cao lớn hớn ở nhóm NV so với CT (64,5\% so với 46,6\%, p = 0,04) (bảng 3). 
Bảng 3. Tỉ lệ tương quan giữa độ tổn thương trên DWI và kêt quả giải phẫu bệnh, giữa độ tổn thương trên DWI với tỉ lệ u bậc cao theo vùng.

\begin{tabular}{|c|c|c|c|c|c|c|}
\hline & \multicolumn{3}{|c|}{$\begin{array}{c}\text { Mối tương quan giữa bậc của u và vị trí u } \\
\text { theo vưng }\end{array}$} & \multicolumn{3}{|c|}{$\begin{array}{l}\text { Tỉ lệ u độ mô học cao theo phân } \\
\text { vừng (pGS } \geq 4+3 \text { ) }\end{array}$} \\
\hline & $\begin{array}{c}\text { NV } \\
(n=76)\end{array}$ & $\begin{array}{c}C T \\
(n=105)\end{array}$ & Giá trị p & $\begin{array}{c}N V \\
(n=76)\end{array}$ & $\begin{array}{c}C T \\
(n=105)\end{array}$ & $\begin{array}{c}\text { Giá trị } \\
\text { p }\end{array}$ \\
\hline $\begin{array}{l}\text { Tống số } \\
\text { III, IV, V }\end{array}$ & $65(87,8 \%)$ & $72(69,9 \%)$ & 0,006 & $49(64,5 \%)$ & $51(48,6 \%)$ & 0,04 \\
\hline $\begin{array}{l}\text { Nghi ngờ } \\
\text { độ III }\end{array}$ & $3(30 \%)$ & $5(33 \%)$ & 1,00 & $0(0,0 \%)$ & $1(6,7 \%)$ & 1,00 \\
\hline $\begin{array}{l}\text { Nghi ngờ } \\
\text { độ IV }\end{array}$ & $11(91,7 \%)$ & $22(64,7 \%)$ & 0,14 & $9(75,0 \%)$ & $14(41,2 \%)$ & 0,09 \\
\hline $\begin{array}{l}\text { Nghi ngờ } \\
\text { độ V }\end{array}$ & $51(98,1 \%)$ & $45(83,3 \%)$ & 0,02 & $40(74,1 \%)$ & $36(63 \%)$ & 0,30 \\
\hline
\end{tabular}

\section{BÀN LUÂ̂N}

Trong nghiên cứu này, chúng tôi quan sát thấy DWI hữu ích trong chẩn đoán UTTTL đối với NV hơn CT. Những bệnh nhân có độ DWI càng cao thì mức độ chẩn đoán chính xác càng cao. Chúng tôi cũng nhận thây DWI âm tính không đảm bảo hoàn toàn không có UTTTL.

Khoảng 70\% đến 75\% UTTTL phát triển từ vùng NV của tuyến tiền liệt, do vậy NV cần được kĩ lưỡng trong thăm khám bằng $\mathrm{CHT}$. DWI thu tín hiệu của các phân tử nước chuyển động ngẫu nhiên, sự chuyển động của các phân tử nước bị cản trở bởi sự tương tác với thành phần mô và màng tế bào. Ở trong mô $T L L$, giá trị $A D C$ tỉ lệ với thể tích khoang và tỉ lệ nghịch với thể tích phân tử. Mật độ tế bào cao hơn với mô UTTTL có điểm Gleason cao và mật độ thấp hơn với điểm Gleason thấp. Do đó, mô u có điểm Gleason cao sẽ hạn chế khuếch tán nhiều hơn so với mô u có điểm mô học thấp. Trong khi đó, một cấu trúc lành tính có mật độ tế bào cao cũng hạn chế khuếch tán có thể gây ra dương tính giả với chẩn đoán UTTTL, ví dụ như nốt phì đại lành tính (PĐLT). Tổn thương PĐLT thường xuất phát từ $\mathrm{CT}$, có thể gây ra DWI dương tính ở CT cao hơn so NV. Điều này góp phần giải thích cho ưu thế của DWI đối với NV so với CT.

Các nốt PĐLT giàu mô đệm khiến cho việc đánh giá UTTTL ở CT khó khăn hơn. Các nốt PĐLT giàu mô đệm giảm tín hiệu trên T2W giống với UTTTL ${ }^{5}$. Nốt PĐLT giàu mô đệm giảm tín hiêu trên $A D C$ do giảm tín hiẹu trên $T 2 W$ và hạn chế khuếch tán do mật độ tế bào cao. Mặc dù PĐLT giàu mô đệm cho giá trị ADC cao hơn UTTTL, sử dung $A D C$ định lượng trong chẩn đoán UTTTL vẫn chưa được áp dưng ${ }^{1}$.

Hiệp hội chẩn đoán hình ảnh niệu-sinh dục châu Âu đưa ra hệ thống phân loại PIRADs (Prostate Imaging Reporting and Data System) lần đầu tiên năm 2012, gần đây đã có phiên bản
2.1 năm 2019 với nhiều thay đổi, đặc biệt trong vai trò của xung DWI trong phân độ tổn thương $\mathrm{NV}$ và $\mathrm{CT}^{1}$. Nghiên cứu của chúng tôi cho thấy DWI đánh giá tổn thương NV tốt hơn tuy nhiên một số bệnh lý khác cũng có thể cho hình ảnh DWI tương tự như viêm tuyến tiền liệt ${ }^{6}$. Trong những trường hợp đó, cần đánh giá xung $T 2 W$ để giảm nguy cơ dương tính giả. Dù kết quả chúng tôi ủng hộ việc sử dụng DWI như xung đầu tay đánh giá tổn thương NV; phối hợp đa chuỗi xung vẫn nên được áp dụng thường quy. Một số báo cáo cho thấy CHT đa chuỗi xung có giá trị dự đoán âm tính cao đối với UTTTL độ mô học cao, đạt gần $100 \%$ đối với bệnh nhân đã được sinh thiết hoăc những người theo dõi có điểm Gleason $6^{7}$. Tuy nhiên, một số khác đưa ý kiến trái chiều, khi phân tích 122 bệnh nhân chụp $\mathrm{CHT}$ đa chuỗi xung trước phẫu thuât, Le và động nghiệp thống kê thây có $20 \%$ khối u bị bỏ sót bởi $\mathrm{CHT}^{8}$. Tương tự, một nghiên cứu trên 101 bệnh nhân cắt tuyển tiền liệt có $\mathrm{CHT}$ trước mồ âm tính, $61,4 \%$ có u với điểm Gleason $\geq 7^{9}$. Trong nghiên cứu này, chúng tôi cũng quan sát thấy một số phần trăm bệnh nhân có u bậc cao mặc dù điểm PIRADs là I-II trên DWI. Những quan sát này cho thấy nguy cơ có u mặc dù DWI âm tính là không thể loại bỏ.

Chúng tôi cũng nhận thấy những giới hạn trong nghiên cứu của mình bao gồm số lượng bênh nhân hạn chế và bản chất nghiên cứu hồi cứu. Tỉ lệ ung thư có nguy cơ cao hơn so với thực tế do chúng tôi chỉ đánh giá được trên những bệnh nhân có kết quả giải phẫu bệnh qua sinh thiết. Nghiên cứu sẽ chính xác hơn khi sử dụng kết quả mổ bệnh phẩm phẫu thuật toàn bộ thay vì kết quả sinh thiết với bệnh nhẩn có DWI âm tính. Đồng thời, nguy cờ điểm của tổn thương Gleason trong nghiên cứu sẽ cao hơn so với thực tế do cách chọn điểm. Trong nghiên cứu này chúng tôi không đánh giá giá trị định tính 
của $A D C$, một thành phần quan trọng của chuỗi xung khuếch tán.

\section{KẾT LUÂ̂N}

Nghiên cứu của chúng tôi chứng minh rằng DWI giúp xác định u ở NV chính xác hơn CT. Trong số những bệnh nhân có PIRADs IV và PIRADs V, DWI chính xác hơn trong xác định UTTTL ở NV hơn là CT, tuy nhiên không có sự khác biệt đáng kể giữa bệnh nhân có độ thấp hơn. Mặc dù vậy, một số lượng không nhỏ bệnh nhân có DWI âm tính nhưng có độ UTTTL cao. Những phát hiện này nên được cân nhắc trong phân tích CHT tuyến tiền liệt xác định UTTTL.

\section{TÀI LIỆU THAM KHẢO}

1. Turkbey B, Rosenkrantz AB, Haider MA, et al. Prostate Imaging Reporting and Data System Version 2.1: 2019 Update of Prostate Imaging Reporting and Data System Version 2. Eur Urol. 2019;76(3):340-351. doi:10.1016/j.eururo.2019.02.033

2. Lee H, Hwang SI, Lee HJ, Byun S-S, Lee SE, Hong SK. Diagnostic performance of diffusionweighted imaging for prostate cancer: Peripheral zone versus transition zone. PLoS One. 2018;13(6):e0199636.

doi:10.1371/journal.pone.0199636

3. Scheenen TWJ, Rosenkrantz AB, Haider MA, Fütterer JJ. Multiparametric Magnetic Resonance
Imaging in Prostate Cancer Management: Current Status and Future Perspectives. Invest Radiol. 2015;50(9):594-600.

doi:10.1097/RLI.0000000000000163

4. Moosavi B, Flood TA, Al-Dandan o, et al, Multiparametric MRI of the anterior prostate gland: clinical-radiological-histopathological correlation. Clin Radiol. 2016;71(5):405-417. doi:10.1016/ j.crad.2016.01.002

5. Akin O, Sala E, Moskowitz CS, et al. Transition zone prostate cancers: features, detection, localization, and staging at endorectal MR imaging. Radiology. 2006;239(3):784-792. doi:10.1148/ radiol.2392050949

6. Polanec S, Helbich TH, Bickel $H$, et al. Headto-head comparison of PI-RADS v2 and PI-RADS v1. Eur J Radiol. 2016;85(6):1125-1131. doi:10.1016/j.ejrad.2016.03.025

7. Wysock JS, Mendhiratta N, Zattoni F, et al. Predictive value of negative $3 \mathrm{~T}$ multiparametric magnetic resonance imaging of the prostate on 12core biopsy results. BJU Int. 2016;118(4):515-520. doi:10.1111/bju.13427

8. Le JD, Tan N, Shkolyar E, et al. Multifocality and prostate cancer detection by multiparametric magnetic resonance imaging: correlation with whole-mount histopathology. EurUrol. 2015;67(3): 569-576. doi:10.1016/j.eururo.2014.08.079

9. Branger $N$, Maubon $T$, Traumann $M$, et al. Is negative multiparametric magnetic resonance imaging really able to exclude significant prostate cancer? The real-life experience. BJU Int. 2017;119(3):449-455. doi:10.1111/bju.13657

\title{
HIÊUU QUẢ ĐIỀU TRỊ CỦA PHÁC Đồ BORTEZOMIB, CYCLOPHOSPHAMIDE VÀ DEXAMETHASONE (VCD) TRÊN BỆNH NHÂN ĐA U TỦY CÓ SUY GIẢM CHỨC NĂNG THÂ̂N
}

\author{
Lê Bảo Ngọc ${ }^{1,2}$, Suzanne Monivong Cheanh Beaupha ${ }^{1,2}$
}

\section{TÓM TẮT}

Đặt vấn đề: Đa u tủy là một bệnh lý ung thư huyết học thường gặp, gây rối loạn chức năng nhiêu cơ quan. Tổn thương thận là một trong những biến chứng phổ biến nhất của bệnh đa u tủy. Nhiều khuyến cáo trên thế giới hiện nay đã lựa chọn phác đồ VCD (bortezomib, cyclophosphamide và dexamethasone) là phác đồ ưu tiên cho bệnh nhân đa u tủy có suy giảm chức năng thận. Tại Việt Nam còn ít nghiên cứu về vấn đề này. Mục tiêu nghiên cứu: Đánh giá hiệu quả điều trị của phác đồ bortezomib, cyclophosphamide và dexamethasone (VCD) trên bệnh nhân đa u tủy mới chẩn đoán có suy giảm chức nẳng

\footnotetext{
${ }^{1}$ Đại học Y Dược TP. Hồ Chí Minh

${ }^{2}$ Bềnh viện Chợ Rẫy

Chịu trách nhiệm chính: Lê Bảo Ngọc

Email: lebaongoc94@gmail.com

Ngày nhận bài: 4.6.2021

Ngày phản biện khoa học: 28.7.2021

Ngày duyệt băi: 5.8.2021
}

thận. Đối tượng và phương pháp nghiên cứu: Đây là nghiên cứu mô tả hồi cứu được thực hiện tại khoa Huyết học bệnh viện Chợ Rẫy trong khoảng thời gian từ năm 2015-2020 ở bệnh nhân từ 18 tuổi trở lên, mới được chẩn đoán đa u tủy và có tăng creatinin máu > $2 \mathrm{mg} / \mathrm{dL}$ hoặc giảm độ lọc cầu thận eGFR < 60 $\mathrm{mL} /$ phút $/ 1.73 \mathrm{~m}^{2}$, được điều trị bằng phác đồ bortezomib, cyclophosphamide và dexamethasone (VCD). Kết quả: Chúng tôi thu thập được 40 bệnh nhân có độ tuổi là $53,4 \pm 9,5$ tuổi, nam giới chiếm $77,5 \%$, creatinin máu là $4,6 \pm 3,2 \mathrm{mg} / \mathrm{dL}$. Sau hoá trị với 4 chu kỳ bằng phác đồ VCD có $77 \%$ bệnh nhân đạt đáp ứng lui bệnh một phần hoặc hơn ( $\geq \mathrm{PR})$, trong đó đạt lui bệnh một phần rất tốt (VGPR) là $27 \%$. Tỷ lệ đáp ứng thận nói chung đạt $85 \%$, trong đó $57 \%$ đáp ứng hoàn toàn (CRrenal), $10 \%$ đáp ứng một phần (PRrenal) và $18 \%$ đáp ứng tối thiểu (MRrenal). Có sự tương quan thuận có ý nghĩa thống kê giữa đáp ứng thân và đáp ứng lui bệnh sau hoá trị với $p=0,002$. Kểt luận: Phác đồ VCD có hiệu quả điều trị cao trong cả đáp ứng lui bệnh và cải thiện chức năng thận trên bệnh nhân đa u tuỷ mới chẩn đoán có suy giảm chức 